\title{
Designing services for frequent attenders to the emergency department: a characterisation of this population to inform service design
}

\author{
Authors: Rebecca Jacob, ${ }^{\mathrm{A}}$ Mai Luen Wong, ${ }^{\mathrm{B}}$ Catherine Hayhurst, ${ }^{\mathrm{C}}$ Peter Watson ${ }^{\mathrm{D}}$ and Cecily Morrison ${ }^{\mathrm{E}}$
}

\begin{abstract}
Frequent attendance to the emergency department (ED) is a growing public health concern. Designing services for frequent attenders poses challenges, given the heterogeneous nature of this group. This was a two-part observational study identifying frequent attenders from ED records. The first stage studied trends and developed personas with emphasis on differentiating moderate frequent attenders (attending between 5 and 20 times per year) and extreme frequent attenders (attending more than 20 times). Stage 2 included a case note review of 100 consecutive frequent attenders. Results showed an increase in frequent attendance from $2.59 \%$ to $4.12 \%$ over 8 years. Moderate frequent attenders accounted for $97 \%$. Of the 100 frequent attenders studied, $45 \%$ had medically unexplained symptoms (MUS), associated with younger age $(p<0.001)$ but not with gender $(p>0.05)$. In conclusion, the ED is a useful hub for identifying frequent attenders with MUS, particularly among moderate frequent attenders; service design for this group should consider a 'whole-systems approach' with integration between primary and secondary care, including specialist liaison psychiatry services where appropriate.
\end{abstract}

Keywords: Frequent attenders, medically unexplained symptoms, MUS, service design

\section{Introduction}

Emergency departments (EDs) in the UK, and indeed internationally, are reporting a steady rise in demand, which they are not resourced to manage. ${ }^{1-3}$ Particular emphasis has been placed on addressing the needs of frequent attenders -

\footnotetext{
Authors: ${ }^{\text {A }}$ consultant psychiatrist, Cambridge and Peterborough NHS Foundation Trust, Cambridge, UK; ${ }^{B}$ associate specialist, Cambridge and Peterborough NHS Foundation Trust, Cambridge, UK; ${ }^{C}$ consultant in emergency medicine, Cambridge University Hospitals NHS Foundation Trust, Cambridge, UK; ' ${ }^{\text {senior }}$ statistician, MRC Cognition and Brain Sciences Unit, University of Cambridge, Cambridge, UK; ${ }^{E}$ research associate, Engineering Design Centre, University of Cambridge, Cambridge, UK
}

those who present to the ED five or more times in a 12-month period - with the hope that this will lead to more cost effective services. ${ }^{3-7}$ Published service models addressing frequent attendance focus on ED-initiated, multidisciplinary 'case management'. 8,9 This involves identifying the patient's medical and social needs and devising implementable strategies to address these, ideally outside an ED environment. This model embodies the common assumption that frequent attenders take up disproportionate amounts of consultation time and would be better served elsewhere, such as primary care or psychiatric services. Evaluations of these models suggest that they can be clinically effective, but their ability to decrease frequent attendance in the long term is unclear; some studies even suggest an increase in attendance..$^{8-10}$

One explanation for these mixed results is that the definition of a frequent attender in these models is imprecise. Indeed, research suggests that frequent attenders are a heterogeneous population who are difficult to characterise ${ }^{3,6,7,11}$ As a starting point to propose alternative service designs, we need a richer characterisation of this group and their healthcare needs. Jelinek et al has made a useful differentiation of frequent attenders, dividing the groups into moderate frequent attenders, who attend between 5 and 20 times per year, and extreme frequent attenders, who attend more than 20 times per year; the latter group includes a population that is more often self-referred, less likely to be hospitalised, and presents with psychosocial problems. ${ }^{12}$ Other studies also showed that the highest frequency users more often present with lower acuity complaints and psychiatric comorbidity. ${ }^{7}$ In contrast, patients with moderate frequent attendance have been shown to present with more circulatory system disorders, greater urgency and a higher admission rate. ${ }^{13-15}$

In this paper, we present two related research studies that describe different aspects of the population who frequently attend Addenbrooke's Hospital, a university teaching hospital in the UK. The first study looks at the extent of frequent attendance. The second study focuses more specifically on the characterisation of frequent attenders; with particular interest in those with medically unexplained and mental health symptoms.

\section{Methods}

The research was carried out under the auspices of the National Institute for Health Research funded Collaborations 
for Leadership in Applied Health Research and Care East of England. This umbrella enabled collaboration between health professionals in the Cambridgeshire Peterborough Foundation Trust, Department of Emergency Medicine at Addenbrookes Hospital, Cambridge University Hospital (CUH) NHS Foundation Trust, and the Engineering Design Centre, at the University of Cambridge.

\section{Study design}

The study conducted was a two-part observational study identifying frequent attenders from the records of those attending frequently to the ED. The first stage studied trends and developed possible personas of the attenders. The second stage was a case note review of 100 consecutive patients attending the ED frequently. According to the policies that constitute research at $\mathrm{CUH}$, the project was submitted for evaluation by the local clinical audit department and was deemed to meet criteria for service evaluation activity, which is exempt from ethics review. Great care was taken to ensure anonymity of patient data; in particular, our case vignettes are anonymised with respect to age and sex.

\section{Stage 1}

Data was extracted retrospectively and anonymised from the Addenbrookes Hospital electronic patient registration system for the time period 1 January 2003 (when the system was put in place) to 31 December 2010. These data included information about gender, age, postcode and primary care trust, and information about the attendance episode and diagnosis. We defined frequent attendance as patients attending more than 5 times in a 12-month period based on previous literature defining this subgroup. ${ }^{3,6}$ We further subdivided this group into moderate frequent attenders, who attend more than 5 and less than 20 times per year, and extreme frequent attenders, who attend more than 20 times per year using previous research definitions. ${ }^{12}$

The stage 1 dataset was anonymised and then analysed using descriptive statistics to help identify frequent attender characteristics and presentation patterns. These results were then augmented to create personas. Personas are a service design tool in which fictional characters are created to represent the different users that might use a product, in this case healthcare provision in the ED. We used previous research findings on frequent attenders to build the characteristics of the persona. ${ }^{16,17}$ Personas play an important role in creating a shared and persistent view of the user, which can be referred to when making design decisions. This is a particularly useful tool when a population's diversity is not actively acknowledged, avoiding problems that arise when people have different ideas of the users and their problems. We used demographics (eg age), behaviour patterns (eg number and time of attendances) and reason for attendance to assist us in defining the personas.

Stage 2

A detailed case note review of a total of 100 consecutive frequent attenders was conducted. In the second stage of data collection, we included all frequent attenders attending 5 or more times per year. The lowering of the threshold from more than 5 attendances/year, used initially, was in order to capture our Stage 1 study findings that the majority of frequent attenders comprised 'moderate frequent attenders' rather than extreme frequent attenders (those who attend more than 20 times per year). ${ }^{13}$

The criterion-based review included assessing demographic details, diagnosis and reason for latest ED attendance, number of attendances to the ED per year, number of specialty outpatient appointments attended, invasive tests and whether there had been at least one mention in the notes of a clinical impression of medically unexplained symptoms (MUS). MUS are defined as physical symptoms, which are inadequately explained, or not at all, by somatic disease. ${ }^{18,19}$ The clinical impression is not a distinct one, often including nebulous physical and psychological ailments on a wide continuum of severity, duration and comorbidity. Common symptoms include chest, abdominal or back pain, tiredness, dizziness, headache, ankle swelling, shortness of breath, insomnia and numbness. ${ }^{20-22}$ MUS are estimated to pose a significant financial cost; one study estimated the cost to the NHS as $\mathfrak{£} 3$ billion per year. ${ }^{22}$

Statistical analysis was conducted to evaluate the relationship between frequent attendance, demographic factors, mental health or MUS and the support mechanisms currently in place for this vulnerable group of patients.

\section{Results}

\section{Stage 1}

\section{Attendances}

Over the 8-year period analysed, 481,372 ED attendances were recorded and yearly data suggests a steady increase in the number of people attending the ED over this time frame. Of these, 20,965 attendances were accounted for by 2,463 individuals; these patients qualified to be included in our definition of a frequent attender (Table 1). The proportion of total attendances by frequent attenders has arisen from $2.59 \%$ in 2003 to $4.12 \%$ of total ED attendances in 2010 . We found that $373(15 \%)$ patients out of 2,463 were repeat frequent attenders (ie attending six times or more in a year in more than 1 year) accounting for nearly half of the attendances between the years 2003 to 2010.

\begin{tabular}{l}
$\begin{array}{l}\text { Table 1. Patients attending more than five times per } \\
\text { year }\end{array}$ \\
\begin{tabular}{lll} 
Year & $\begin{array}{l}\text { Total number } \\
\text { of patients, } \mathbf{n}\end{array}$ & $\begin{array}{l}\text { Number of patients } \\
\text { who are FA, } \mathbf{n}(\%)\end{array}$ \\
2003 & 50,122 & $186(0.37)$ \\
2004 & 53,540 & $199(0.37)$ \\
2005 & 54,735 & $244(0.45)$ \\
2006 & 56,931 & $260(0.46)$ \\
2007 & 62,962 & $323(0.51)$ \\
2008 & 64,867 & $367(0.57)$ \\
2009 & 68,556 & $432(0.63)$ \\
2010 & 69,659 & $452(0.65)$ \\
Total & 481,372 & $2,463(0.51)$ \\
\hline FA frequent attenders.
\end{tabular} \\
\hline
\end{tabular}




\begin{tabular}{|c|c|c|c|c|c|}
\hline Year & $\begin{array}{l}\text { MFA 5-10 } \\
\text { visits }\end{array}$ & $\begin{array}{l}\text { MFA 10-20 } \\
\text { visits }\end{array}$ & $\begin{array}{l}\text { EFA 20-30 } \\
\text { visits }\end{array}$ & $\begin{array}{l}\text { EFA >30 } \\
\text { visits }\end{array}$ & Total \\
\hline 2003 & 1087 & 407 & 43 & 68 & 1605 \\
\hline 2004 & 1278 & 246 & 49 & 0 & 1573 \\
\hline 2005 & 1432 & 434 & 200 & 0 & 2066 \\
\hline 2006 & 1571 & 444 & 166 & 142 & 2323 \\
\hline 2007 & 1837 & 652 & 146 & 247 & 2882 \\
\hline 2008 & 2181 & 615 & 207 & 150 & 3153 \\
\hline 2009 & 2686 & 526 & 240 & 106 & 3558 \\
\hline 2010 & 2652 & 863 & 185 & 105 & 3805 \\
\hline Total & 14724 & 4187 & 1236 & 818 & 20965 \\
\hline
\end{tabular}

$\mathrm{EFA}=$ extreme frequent attendance; $\mathrm{MFA}=$ moderate frequent attendance

\section{Moderate and extreme frequent attenders}

Table 2 breaks down the data into number of moderate frequent attenders and extreme frequent attenders. Each year $97 \%$ of patients fall into the moderate frequent attenders group; they account for $90.5 \%$ of attendances by frequent attenders.

Proposed mental health personas for frequent attenders To postulate personas with possible mental health-related complaints, we combined both our specialist knowledge, previous research on frequent attenders, and the characteristics and presentation patterns identified. We developed three 'personas' of interest:

1 Moderate frequent attender with undiagnosed MUS or somatoform disorders.

2 Moderate frequent attender as a result of long-term condition(s).

3 Extreme frequent attender usually attending with substance misuse or alcohol problems associated with self-harm or mental illnesses.

\section{Stage 2}

\section{Demographic data and characteristics}

The age range of the sample was 17-95 years; the median age was 32 years. The majority of patients were white British, reflecting the local population. Of the patients included in the case note review, 65\% (65/100) had mental health symptoms; $71 \%$ of these $(32 / 65)$ also had mental health problems. Of the overall group, $15 \%$ (15/100) had significant alcohol problems. All patients with alcohol problems in our sample also had mental health difficulties.

\section{Frequent attenders and personas}

Fourteen out of the 100 cases studied were extreme frequent attenders. As postulated in our 'persona types', extreme frequent attenders were significantly more likely than moderate frequent attenders to have alcohol problems $(5 / 14, \mathrm{p}=0.03)$ and mental health conditions $(13 / 14, \mathrm{p}=0.018)$, and less likely to have evidence of a medical/physical diagnosis $(5 / 14, \mathrm{p}=0.036)$.

\section{Medically unexplained symptoms}

Of the patients included in the case note review, 45\% (45/100) had MUS. This included a clinical diagnosis of any symptom recorded by clinicians in the medical notes as lacking physical or test correlates. For example, patients were described on occasion as having MUS per se but they were also reported to have 'functional somatic syndromes,' such as chronic fatigue, non-cardiac chest pain and non-epileptic seizures. Common symptoms of those presenting to the ED with an associated diagnosis of MUS included abdominal pain, chest pain, shortness of breath and dizziness. The mean age of patients with MUS was 36.8 years. MUS were associated significantly with a younger age $(p<0.001)$ but not with gender $(p>0.05)$. Older age patients were more likely to have positive test results or medically 'explained' symptoms $(\mathrm{p}=0.004)$.

\section{MUS and frequency of attendance}

The number of ED attendances was not significantly different for those with versuswithout MUS. However, as suggested by previous research, MUS were largely represented by moderate frequent attenders $(\mathrm{p}<0.001)$, that is, those who attend more between 5 and 20 times per year.

\section{MUS and service provision}

Of the patients with MUS, 91\% (41/45) had invasive tests or procedures requested by multiple specialties. All patients with MUS were also seeing more than one specialty and on average had been seen by five specialist (secondary care) teams. This suggests that patients with frequent attendance to the ED make frequent use of other health services.

Of patients with MUS, 71\% (32/45) also had mental health symptoms and of these, $47 \%(15 / 32)$ had mental health input from secondary providers. There was no evidence of a whole systems approach in the care of this group despite a recent report offering guidance for commissioning integrated urgent and emergency care. ${ }^{5}$ Two of the 45 patients (4\%) had input addressing MUS; this included specialist psychiatric or psychological support from liaison psychiatry services to address MUS symptoms specifically.

\section{Case studies}

Medically unexplained symptoms

Patient A, presented to the ED nine times in the past 12 months. The symptoms related to ED presentation included back pain and shortness of breath. There was no past psychiatric history, although the notes suggest that A had been under stress in recent months.

Over the years, A had been referred either by the ED or the GP to cardiology, rheumatology, medicine, trauma, respiratory and infectious disease outpatient departments. A had also undergone multiple tests, including X-rays, echocardiography and magnetic resonance imaging, which were all unremarkable. A had been diagnosed by the infectious diseases department to have 'post viral fatigue' and the cardiologists had documented 'non-cardiac chest pain', terminology used to denote functional somatic syndromes.

\section{Medically explained symptoms (long-term conditions)}

Patient B presented to the ED seven times in a year for recurrent falls and urinary tract infection; B was often admitted 
following ED presentations. B's GP had been treating B for mild anxiety symptoms with antidepressants but B had not been seen by secondary mental health services.

$B$ had been referred by either the ED or their GP to geriatric medicine, dermatology, rheumatology and general medicine. Multiple invasive tests had been performed with a number of positive findings.

B had multiple medical diagnoses, including squamous cell carcinoma of the hand, polymyalgia, cerebrovascular disease, giant cell arteritis and ischemic heart disease.

Medically explained and unexplained symptoms

Patient $\mathrm{C}$ was noted to have $13 \mathrm{ED}$ attendances in the last year and had been referred by their GP or the ED to surgery, ophthalmology, hepatology and gastroenterology.

$\mathrm{C}$ had been given a diagnosis of cholecystitis and had had a cholecystectomy in the year prior to the frequent ED attendances; $\mathrm{C}$ had also had multiple invasive tests following surgery that were within normal limits.

Postoperatively, C presented with multiple medically unexplained symptoms including chest pain, abdominal pain, double vision and headache. Gastroenterologists had given $\mathrm{C}$ a diagnosis of irritable bowel syndrome and felt the abdominal pain was 'medically unexplained'.

\section{Discussion}

ED services are experiencing a steady increase in attendances, including those with multiple presentations and diverse healthcare needs. This study explores the extent to which frequent attendance is evident in the ED of a large teaching hospital and the trends, characteristics and needs of this subset of patients. The overall increase in the number of attendances, as well as frequent attendance, is in keeping with those reported by the Department of Health for England. ${ }^{24}$

A number of our findings are helpful to take into account when planning services and improving care for frequent attenders to the ED. Firstly, frequent attenders do indeed constitute a heterogeneous population and service design must take into account the fact that 'one size will not fit all'. Moderate frequent attenders and those who are extreme frequent attenders, have different patterns of attendance and service needs. We also noted that among the subgroup of moderate frequent attenders, some patients have medically 'unexplained' symptoms, others medically 'explained' ones and some have both, highlighting the intertwined association between many physical and medical conditions. ${ }^{5}$

It appears that the ED is a useful hub for identifying patients suffering with MUS. The findings relating to the significant likelihood of frequent attenders with MUS being younger and not showing a predisposition to any specific gender, is contrary to previous research suggesting that risk factors relate to older age and female gender. ${ }^{19}$ Perhaps this can be explained by the fact that previous studies relate to routine primary and secondary clinical care as opposed to the frequent presentations to the ED. ${ }^{20,22}$ Additionally, MUS sufferers in this sample were referred to at least one clinic with the majority seeing five specialties for their symptoms, possibly explaining previous research related to significant cost utilisation in this group. Our findings are also contrary to previous research findings that suggested somatisation in the ED represent only a small group of frequent attenders and are not more common than in 'routine attenders'. ${ }^{25}$ There are a number of possible explanations to this anomaly. It might be that, as services have changed, those who attended primary care in the past are now attending the EDs instead. It is also possible that previous research focused more on extreme frequent attenders whereas our findings suggest MUS patients are significantly more likely to be among the moderate frequent attenders.

Integrating services for MUS at the ED interface may be useful to capture this patient population. Furthermore, given that moderate frequent attenders constitute at least $97 \%$ of the frequent attender population locally, collaborative healthcare planning in the design and delivery of a proactive service that offers identification, specialist assessment and appropriate signposting/treatment for this group will likely deliver the highest returns on investment. We believe this will work best as an agedefined pathway focused on adults of working age (eg 16-65).

Our findings must be viewed in light of the limitations imposed: chiefly, the fact that the methodology included a retrospective case note review. In this context, full psychosocial assessments of each frequent attender considered to have MUS could not be carried out; an assessment that could more accurately inform diagnoses and future service design. Additionally, this is a study performed at a single ED and the results are not necessarily generalisable to other EDs.

Notwithstanding these limitations, our conclusions suggest that despite growing interest from many areas of healthcare in frequent attenders, MUS and long-term conditions, current service models are not oriented towards the largest group of frequent attenders. As such, the needs for the above clusters of high demand patients are not being addressed in a joinedup manner using a whole-systems approach (joint working between local authorities, primary and secondary care, as well as acute and liaison psychiatry). This study provides initial guidance for service design efforts in this area.

\section{Conflicts of interest}

All authors declare no conflicts of interest.

\section{Acknowledgments}

This paper was supported by the National Institute for Health Research (NIHR) Collaboration for Leadership in Applied Health Research and Care, East of England (CLAHRC EOE). The views expressed are those of the authors and not necessarily those of the NHS, the NIHR or the Department of Health.

We are grateful to Professor Peter Jones and Dr Christine Hill, director and deputy director of CLAHRC East of England; Associate Professor Belinda Lennox, deputy director CLAHRC Oxford; Dr Cathy Walsh, clinical director of specialist services at Cambridge and Peterborough NHS Foundation Trust; and clinical staff in the emergency department at Addenbrookes Hospital Cambridge, for their support.

\section{References}

1 Boyle A, Bemiuk K, Higginson I, Atkinson P. Emergency department crowding: time for interventions and policy evaluations. Emerg Med Int 2012;2012:838610.

2 Appleby J. Pressures on accident and emergency services. London: The King's Fund, 2013. Available at http://www.kingsfund.org.uk/ audio-video/john-appleby-pressures-accident-and-emergencyservices [Accessed 22 April 2016]. 
3 LaCalle E, Rabin E. frequent users of emergency departments: the myths, the data, and the policy implications. Ann Emerg Med 2010;56:42-8.

4 Cooke M, Fisher J, Dale J et al. Reducing attendances and waits in emergency departments - a systematic review of present innovations. London: NCCSDO, 2004.

5 Fernandes A. Guidance for commissioning integrated urgent and emergency care: a 'whole system' approach. London: RCGP Centre for Commissioning, 2011.

6 Wooden MD, Air TM, Schrader GD, Wieland B, Goldney RD. Frequent attenders with mental disorders at a general hospital emergency department. Emerg Med Australas, 2009;21:191-5.

7 Moore L, Deehan A, Seed P, Jones R. Characteristics of frequent attenders in an emergency department: analysis of 1-year attendance data. Emerg Med J 2009;26:263-7.

8 Skinner J, Carter L, Haxton C. Case management of patients who frequently present to a Scottish emergency department. Emerg Med J 2009;26:103-5.

9 Phillips GA, Brophy DS, Weiland TJ, Chenhall AJ, Dent AW. The effect of multidisciplinary case management on selected outcomes for frequent attenders at an emergency department. Med J Aust 2006;184:602-6.

10 Soril LJJ, Leggett LE, Lorenzetti DL, Noseworthy TW, Clement FM. Reducing frequent visits to the emergency department: a systematic review of interventions. PLoS One 2015;10:1-18.

11 Hodgson P, Smith P, Brown T et al. Stories from frequent attenders: a qualitative study in primary care. Ann Fam Med 2005;3:318-23.

12 Jelinek GA, Jiwa M, Gibson NP, Lynch AM. Frequent attenders at emergency departments: a linked-data population study of adult patients. Med J Aust 2008;189:552-6.

13 Wong ML, Morrison C, A service design approach to frequent attendance in the emergency department. Cambridge: NIHR CLAHRC for Cambridgeshire \& Peterborough; 2012. Available at http:// clahrc-cp.nihr.ac.uk/wp-content/uploads/2012/12/CLAHRC-MUS_ briefing-final-rev291212.pdf [Accessed 22 April 2016].

14 Locker TE, Baston S, Mason SM, Nicholl J. Defining frequent use of an urban emergency department. Emerg Med J 2007;24:398-401.

15 Byrne M, Murphy AW, Plunkett PK et al. Frequent attenders to an emergency department: a study of primary health care use, medical profile, and psychosocial characteristics. Ann Emerg Med 2003;41:309-18.
16 Calabria T. An introduction to personas and how to create them. Broadway: Step Two, 2004. Available at www.steptwo.com.au/papers/ kmc_personas/ [Accessed 22 April 2016].

17 Le Rouge C, Ma J, Sneha S, Tolle K. User profiles and personal in the design and development of consumer health technologies. Int J Med Inform 2013;82:e251-68.

18 Creed F, Henningsen P, Fink P. Medically unexplained symptoms, somatisation and bodily distress: developing better clinical services. Cambridge: Cambridge University Press, 2011.

19 Mangwana S, Burlinsons S, Creed F. Medically unexplained symptoms presenting at secondary care. Int $J$ of Psychiatry Med 2009;39:33-44.

20 Salmon P, Ring A, Humphris GM, Davies JC, Dowrick CF. Primary care consultations about medically unexplained symptoms: how do patients indicate what they want? J Gen Intern Med 2000;24:450-6.

21 Dirkzwager A, Verhaak P. Patients with persistent medically unexplained symptoms in general practice: characteristics and quality of care. BMC Fam Pract 2007;8:33.

22 Curtis P. 'Frequent flyers' costing NHS £2.3bn a year. London: The Guardian, 2006.

23 Nimnuan C, Hotopf M, Wessley S. Medically unexplained symptoms: an epidemiological study in seven specialities. J Psychosom Res 2001;51:361-7.

24 Department of Health. A \& E clinical quality indicators: implementation guidance and data definitions. London: DoH, 2010. Available at http://webarchive.nationalarchives.gov.uk/20130107105354/ http:/www.dh.gov.uk/en/Publicationsandstatistics/Publications/ PublicationsPolicyAndGuidance/DH_122868 [Accessed 22 April 2016].

25 Williams ERL, Guthrie E, Mackaway-Jones K et al. Psychiatric status, somatisation and health care utilisation of frequent attenders at the emergency department: a comparison with routine attenders. J Psychosom Res 2001;50:161-7.

Address for correspondence: Dr R Jacob, Fulbourn Hospital, Fulbourn, Cambridge CB21 5BE, UK.

Email: Rebecca.Jacob@cpft.nhs.uk 